\title{
Development of Photon Detectors for a Fast Focusing DIRC
}

\author{
C. Field, T. Hadig, David W.G.S. Leith, G. Mazaheri, B. Ratcliff, \\ J. Schwiening, J. Uher, ${ }^{+}$and J. Va'vra ${ }^{*}$ \\ SLAC, Stanford University, CA 94309, U.S.A.**
}

\begin{abstract}
We report progress in developing a Focusing DIRC with very good timing resolution. This basic detector development has been motivated by a possible upgrade of the very successful BaBar DIRC particle identification detector for a future Super B-factory. We have built a single bar full size prototype, which aims to reduce the chromatic error by precise timing, and to remove the effect of bar thickness with a focusing mirror. This paper describes the design of the prototype, and systematic studies of the timing resolution and position response for single photons for two 64-pixel detectors: (a) Hamamatsu Flat Panel PMTs, and (b) Burle MCP-PMTs. To test the prototype, we have developed new electronics for $\sim 300$ pixels capable of measuring a single electron timing resolution to $\sim 100 \mathrm{ps}$. We also report on a first measurement of aging with the MCP-PMT.
\end{abstract}

Keywords: Photodetectors; Cherenkov detectors; RICH.

\section{INTRODUCTION}

The DIRC detector at the BaBar experiment provided excellent particle identification performance $[1,2]$. Because of this success, our group is attempting to develop a next generation DIRC, which is capable of not only measuring an $\mathrm{x} \& \mathrm{y}$ coordinate of each photon with similar angular resolution to the present BaBar DIRC, but, in addition, each photon's time-of-propagation (TOP ${ }^{1}$ ) through the Fused Silica bar with $\sim 100$ ps timing resolution (the present BaBar DIRC has a timing resolution of only $\sigma \sim 1.6 \mathrm{~ns}$ ). This new capability will allow a correction of the chromatic error by timing. In addition, the precise timing will help to suppress the background, which would be probably necessary at any future Super B-factory. The focusing element will also remove the bar thickness from the resolution consideration. The smaller pixel size will reduce the overall size of the photon detector, ${ }^{2}$ which will also help to reduce the background .

A DIRC-like detector equipped with the modern fast photon detectors appears to be the only PID system capable of correcting out the chromatic error, at least in principle. To do that one needs to achieve $100-200$ ps timing resolution per single photon ${ }^{3}$.

The present efforts of our group are aiming to develop a reliable photon detector, learn how to achieve $\sim 100$ ps single photon timing resolution on a scale of $\sim 300$ channels, and decide how to best develop electronics on much larger scale for the future. We have built a full length, single bar prototype that will be

* Visiting from the Institute of Experimental and Applied Physics, Czech Technical University, CZ-12800 Prague 2, Czech Republic.

* Corresponding author, tel.1-650-926-2658, e-mail: jjv@slac.stanford.edu.

** Work supported by the Department of Energy, contract DEAC02-76SF00515.

Definition: $\operatorname{TOP}\left(\Phi, \theta_{\mathrm{c}}, \lambda\right)=\left[\mathrm{L} / \mathrm{v}_{\mathrm{g}}(\lambda)\right] \mathrm{q}_{\mathrm{z}}\left(\Phi, \theta_{\mathrm{c}}\right), \theta_{\mathrm{c}}-$ Cherenkov angle, L - distance of light travels in the bar, $\mathrm{v}_{\mathrm{g}}(\lambda)$ - group velocity of light, $\lambda$ - photon wavelength, and $\mathrm{q}_{\mathrm{z}}\left(\Phi, \theta_{\mathrm{c}}\right)$ - z-component of the unit velocity vector.

In BaBar DIRC, this includes a standoff box (SOB) containing $\sim 6000$ liters of water to provide optical coupling between the bars and $\sim 11,000$ PMTs [1].

At this level of resolution the time coordinate does not usually provide a competitive angular measurement with that provided by the $\mathrm{x} \& \mathrm{y}$ coordinates. 
tested in the beam at SLAC soon. The prototype has a single DIRC bar of $\sim 3.6$ meters length, and a focusing element made of a $50 \mathrm{~cm}$ focal length spherical mirror placed in a small optical box filled with mineral oil, which is the coupling medium between the bar and six 64-pixel photon detectors. The system is instrumented with $\sim 300$ channels of electronics.

(a)

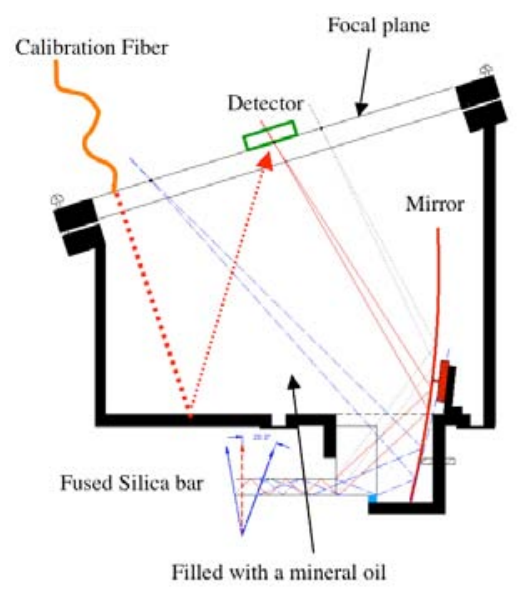

(c)

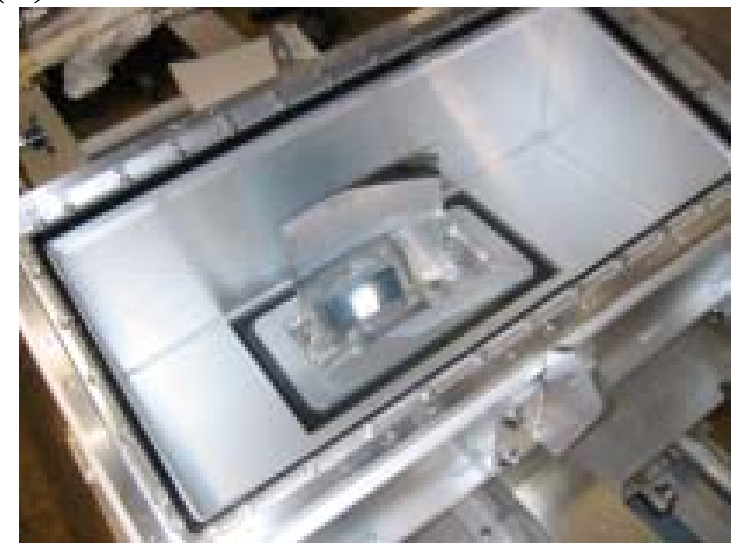

(b)

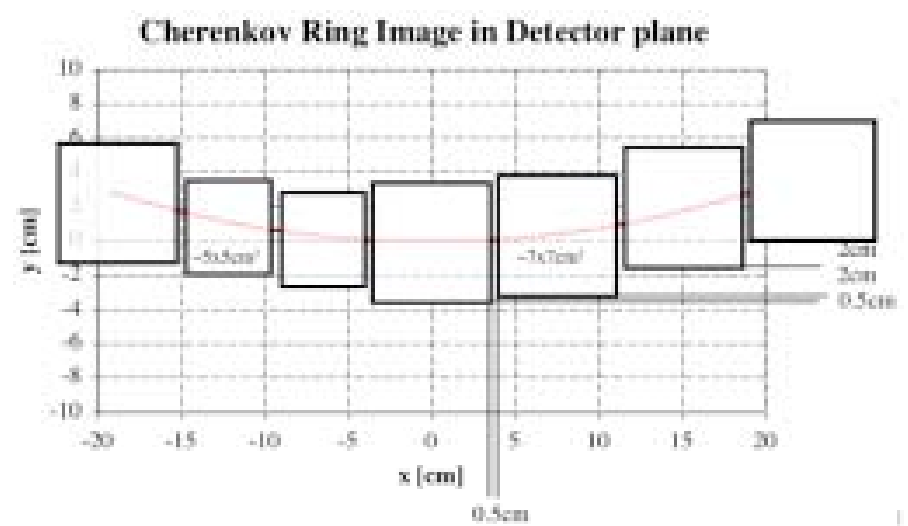

(d)

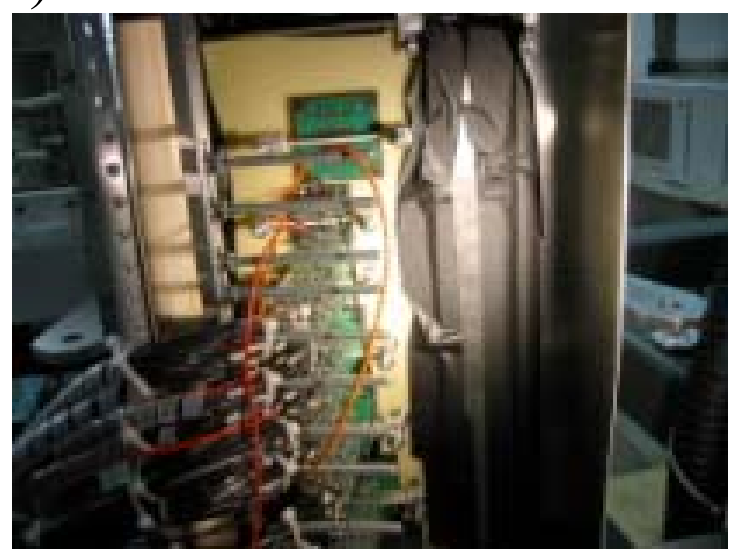

Fig. 1. (a) Principle of the Focusing DIRC prototype. (b) The photon detector arrangement in the focal plane. (c) Optical box, filled with the mineral oil, shows a spherical mirror . (d) Electronics and the photon detectors.

\section{PROTOTYPE DESCRIPTION}

Figure 1 shows the concept and practical realization of the Fast Focusing DIRC prototype. This prototype is the first version of an "Fast Focusing DIRC" [3], however, it will not work in a magnetic field as yet, and the volume between the bar and the photon detectors is simply filled with mineral oil, instead of an expensive solid Fused Silica focusing piece, which would provide higher transparency near 300nm. Fig. 1a shows how the prototype's spherical mirror is designed to remove the effect of bar thickness on the resolution. The optics defines a focal plane where the photon detectors are located. The prototype's photon detection is based on six 64-pixel photon detectors, four Burle MCP-PMTs, and two Hamamatsu Flat-panel MaPMTs. One should add that the prototype was designed to study the chromatic effects in the beam, and no effort was made to optimize it for any real application as a particle identification device. Fig. 1a also shows schematically a calibration system for the detectors using PiLas laser diode. The prototype is on the 
beam line at SLAC, being tested with PiLas laser diode light pulses. It should be ready to take beam data sometime during the spring of 2005.

(a)

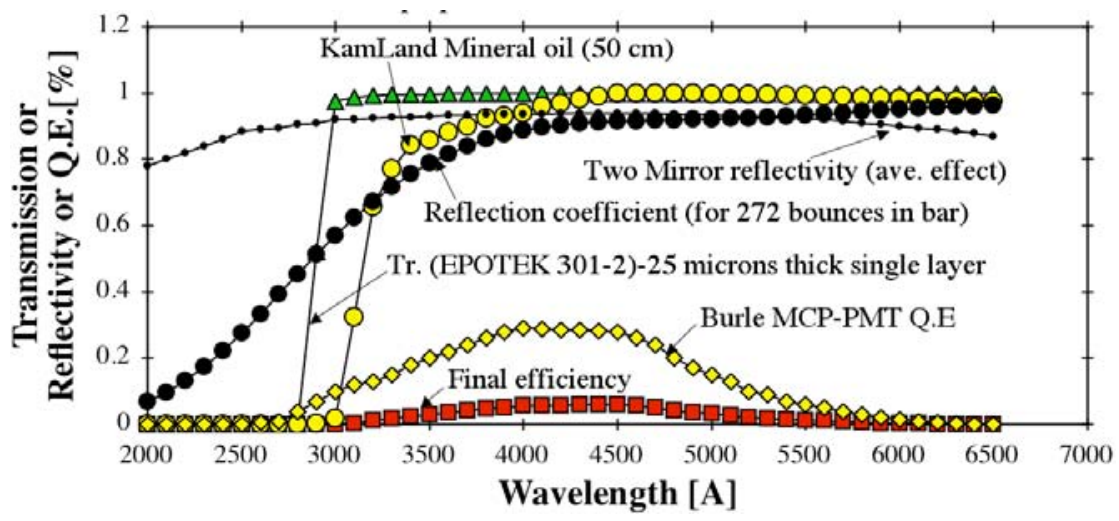

(b)

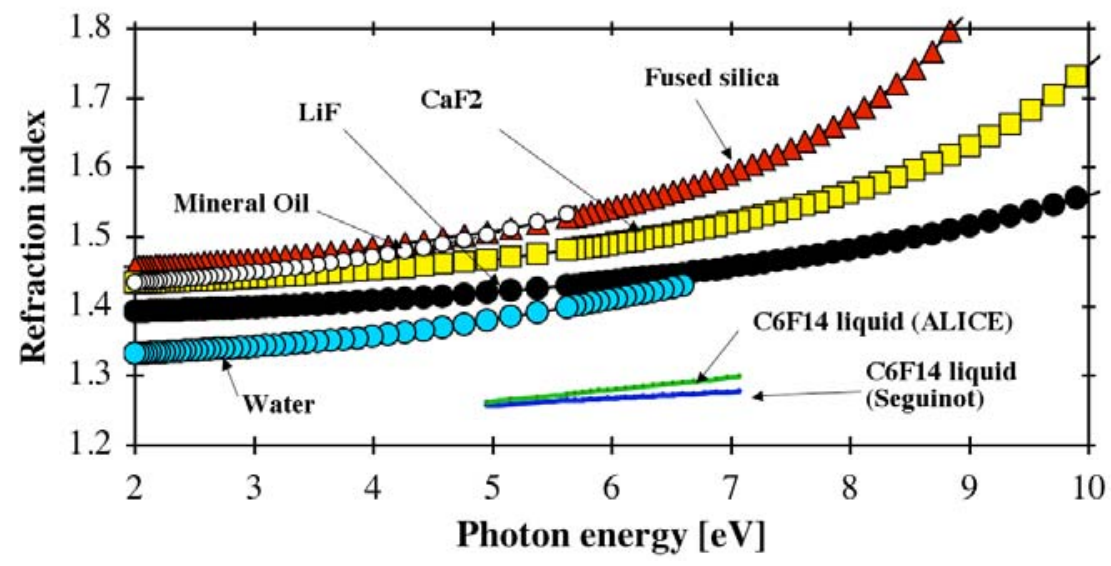

(c)

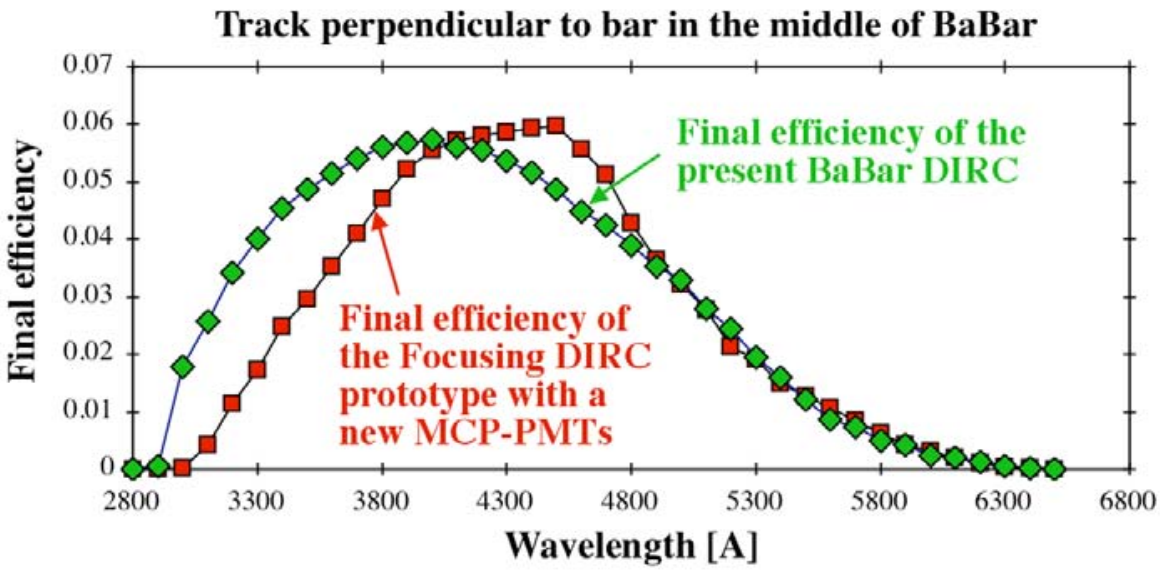

Fig. 2. (a) Various efficiencies in the Focusing DIRC prototype if placed into BaBar, assuming that we take Burle MCP-PMT quantum efficiency [4]. (b) Refraction index of several materials, including water, mineral oil and Fused Silica. (c) An estimate of the relative final detection efficiency of the Focusing DIRC prototype and the present BaBar DIRC, assuming that we would build the Focusing DIRC according to Fig. 1, i.e., including the KamLand mineral oil transmission, Burle's recently quoted MCP-PMT QE, and the best present estimate of future MCP-PMT collection and boundary efficiencies by Burle (see Table 1). 
Figure $2 \mathrm{a}$ shows various efficiencies ${ }^{4}$ for a perpendicular track entering the Fast Focusing DIRC prototype in the middle of its acceptance, if "it would be placed into BaBar" [4]. In this design, the detector optical box is filled with mineral oil from the KamLand experiment, which simplifies the construction and makes it affordable at this particular stage. This is not our first choice, as the mineral oil does not have as good transmission near $300 \mathrm{~nm}$ as, for example water. ${ }^{5}$ On the other hand, its refraction index is a better match to Fused silica, as one can see in Fig. 2b. Fig. 2c shows an estimate of the relative final detection efficiency of the Fast Focusing DIRC prototype and the present BaBar DIRC, assuming the KamLand mineral oil transmission, the Burle's recently quoted MCP-PMT QE, and the best present estimate of future MCP-PMT collection and boundary efficiencies by Burle Co. Ultimately, we would propose to construct a Fast Focusing DIRC similar to Fig. 3 [3]. Table 1 compares expected contributions to the Cherenkov angle resolution of the present BaBar, the Fast Focusing DIRC prototype, and the final Fast Focusing DIRC [3].

Table 1: Expected contributions to the Cherenkov angle resolution from various variables:

\begin{tabular}{|l|c|c|c|}
\hline $\begin{array}{l}\text { Contribution to } \\
\text { Cherenkov angle } \\
\text { resolution [mrads] }\end{array}$ & Present BaBar & $\begin{array}{l}\text { Focusing DIRC } \\
\text { prototype }\end{array}$ & $\begin{array}{l}\text { Ultimate DIRC } \\
\text { of the future }\end{array}$ \\
\hline$\Delta \theta_{\text {track }}$ & $\sim 1$ & $\sim 1$ & $\sim 1$ \\
\hline$\Delta \theta_{\text {chromatic }}$ & $\sim 5.4$ & $\sim 1$ & $\sim 1$ \\
\hline$\Delta \theta_{\text {transport along the bar }}$ & $2-3$ & $2-3$ & $\sim 1$ \\
\hline$\Delta \theta_{\text {bar thickness }}$ & $\sim 4.1$ & $\sim 1$ & $\sim 1$ \\
\hline$\Delta \theta_{\text {PMT pixel size }}$ & $\sim 5.5$ & $\sim 4$ & $\sim 1$ \\
\hline$\Delta \theta_{\mathrm{c}}^{\text {track }}$ & $\sim 2.4$ & $\sim 1.5$ & $\sim 1$ \\
\hline Total $\Delta \theta_{\mathrm{c}}^{\text {photon }}$ & $\sim 9.6$ & $\sim 4.8$ & $2-3$ \\
\hline
\end{tabular}
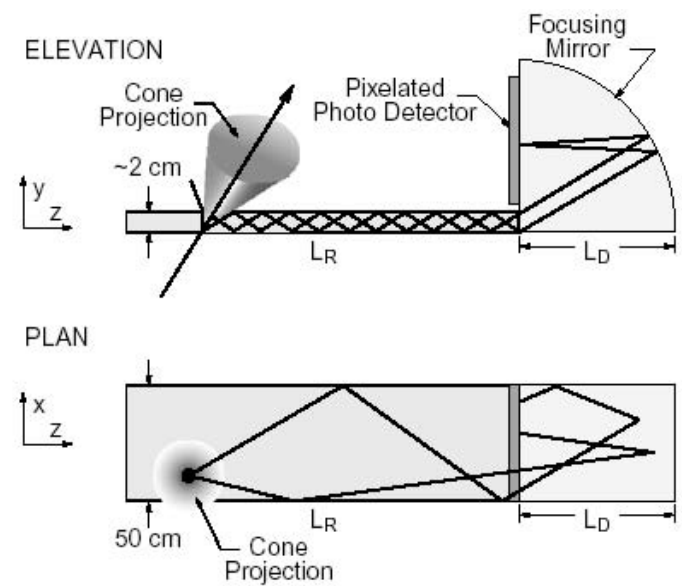

Fig. 3. Plan for the future: the Fast Focusing DIRC concept for the Super B factory [3].

\footnotetext{
${ }^{4}$ With a new MCP-PMT according to Table 2, we estimate $\mathrm{N}_{\mathrm{o}} \sim 29 \mathrm{~cm}^{-1}$, and $\mathrm{N}_{\mathrm{pe}} /$ track $\sim 27$ for $\Theta_{\text {track }}=90^{\circ}$ in the middle of the acceptance.

${ }^{5}$ One should point out, however, that no attempt was made to clean this oil in any special way, and further transmission improvements are likely.
} 
(a)

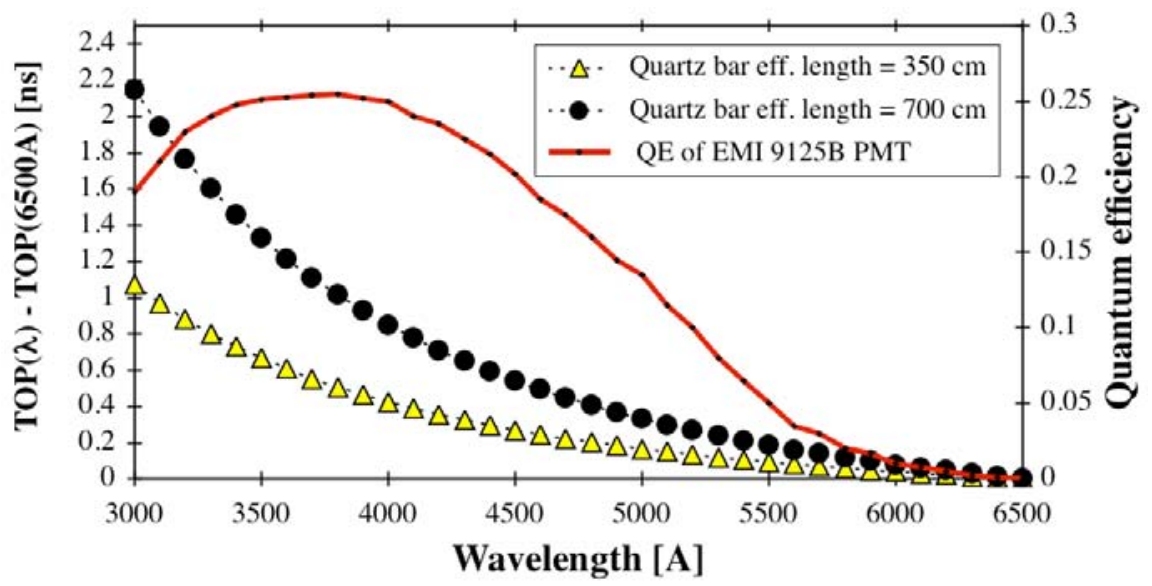

(b)

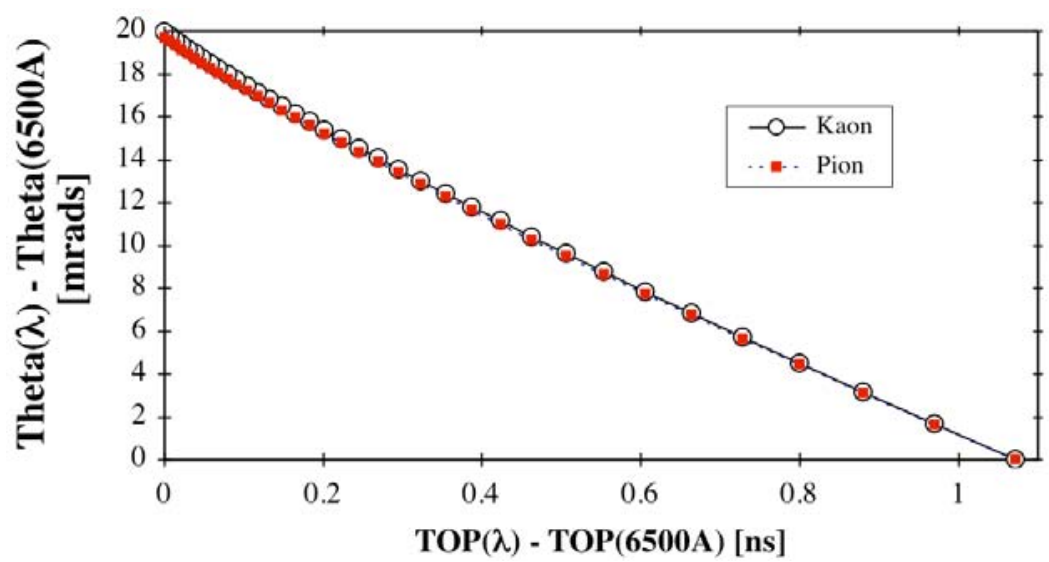

Fig. 4. (a) Timing shift from the chromaticity in a long DIRC bar as a function of the photon wavelength [4]. The simplified calculation assumes that the track is perpendicular to bar. It considers only photons propagating in y-z plane; the track momentum is $4 \mathrm{GeV} / \mathrm{c}$, and the bar effective length is either 7 or 3.5 meters depending where the particle enters the bar. The Focusing DIRC prototype is $\sim 3.6 \mathrm{~m}$ long, therefore, we expect up to $\sim 2 \mathrm{~ns}$ timing shift across the bandwidth of the Bialkali photocathode. A timing resolution at a level of 100-200ps $\sigma$ will be able to start measuring this effect. (b) Correlation between the chromaticity of the TOP variable and the corresponding chromatic change in the Cherenkov angle.

Timing dependence on the chromaticity in a 3.6-meter long DIRC bar is shown in Fig. 4a for a track entering the bar perpendicularly [4]. We expect up to $\sim 2$ ns timing shift due to chromaticity, depending where the particle enters the bar, and given the wavelength bandwidth of the Bialkali photocathode. Timing resolution at a level of 100-200ps will be able to determine this quite well. One should add that the TOP variable and the Cherenkov angle correlate with each other over the chromatic bandwidth - see Fig 4b.

Based on the values in Table 1, one can predict the expected pi/K separation in the Fast Focusing DIRC prototype and compare it with the performance expected in BaBar DIRC and the final Fast Focusing DIRC - see Fig. 5. The assumptions which went into this calculation were: (a) in case of the Focusing DIRC, we assume $\sim 6 \mathrm{~mm}^{2}$ pixel size, completely corrected chromatic error, optics to remove the bar thickness, no loss of photons in the photon detectors, and (b) in case of the ultimate DIRC, we assume, in addition, an "infinitely precise" photon detector and additional improvements in the tracking. Clearly, the detector 
assumptions in both cases are not $100 \%$ realistic, and therefore one should take Fig. 5 as an illustration only. A full scale MC is needed.

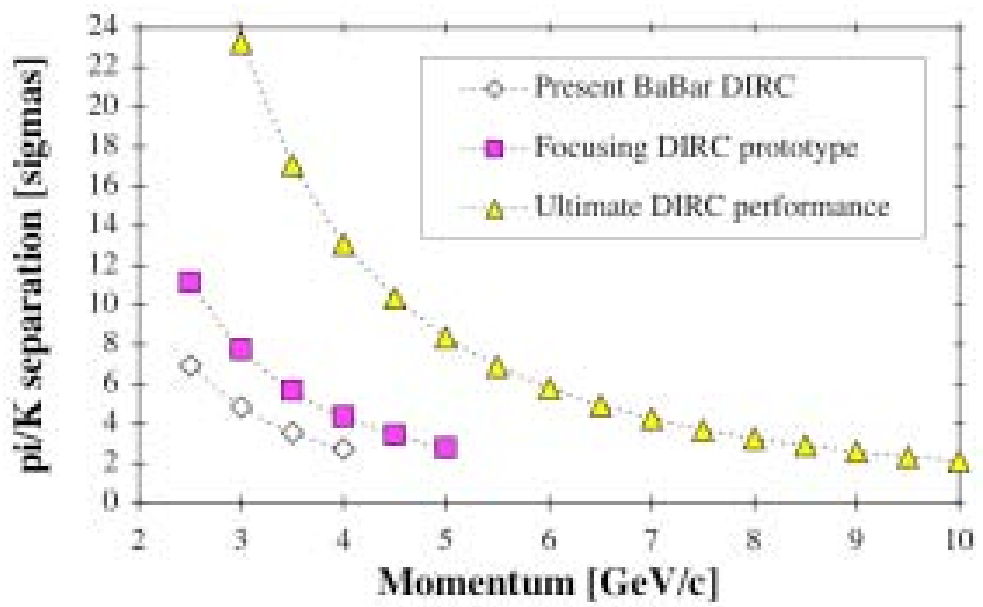

Fig. 5. The expected pi/K separation based on Table 1 for the present BaBar DIRC, the Fast Focusing DIRC prototype, and a final Fast Focusing DIRC [3].

The detectors under study presently for the Focusing DIRC prototype are various versions of Burle MCP-PMTs and Hamamatsu MaPMTs, each having 64 pixels. Table 2 shows the present and future specifications of the Burle detector. One should note that the total fraction of "in time" photoelectrons is rather low, $\sim 50 \%$ even in the best case in future tubes. Table 3 shows similar data for the Hamamatsu MaPMT. One should add a small comment that we are perfectly open to other new detector ideas, if proven to be practical, such as SiPM detectors [5].

Table 2: Burle MCP-PMT specifications:

\begin{tabular}{|l|c|c|c|}
\hline Parameter & H85011-501 & H85011-403 & New tube \\
\hline Photocathode type & Bialkali & Bialkali & Bialkali \\
\hline Number of MCPs per PMT & 2 & 2 & 2 \\
\hline Total average gain @ -2.3kV & $\sim 5 \times 10^{5}$ & $\sim 5 \times 10^{5}$ & $\sim 10^{6}$ \\
\hline MCP hole diameter & $25 \mu \mathrm{m}$ & $25 \mu \mathrm{m}$ & $10 \mu \mathrm{m}$ \\
\hline MCP hole angle relative to perpendicular & $12^{\circ}$ & $12^{\circ}$ & $12^{\circ}$ \\
\hline Geometrical collection efficiency of the 1-st MCP & $60-65 \%$ & $60-65 \%$ & $70 \%$ \\
\hline Geometrical packing efficiency (for raw tube) & $67 \%$ & $67 \%$ & $85 \%$ \\
\hline Total fraction of “in time" photoelectrons detected & $30-35 \%$ & $30-35 \%$ & $\sim 50 \%$ \\
\hline SLAC measurement of single electron resolution $\sigma_{\text {narrow }}$ & $50-70 \mathrm{ps}$ & $50-70 \mathrm{ps}$ & - \\
\hline Amplifier used in SLAC measurement & Elantec $2075 \mathrm{C}$ & Elantec $2075 \mathrm{C}$ & - \\
\hline Voltage gain of SLAC amplifier & $\sim 130 \mathrm{x}$ & $\sim 130 \mathrm{x}$ & - \\
\hline Matrix of anode pixels & $8 \times 8$ & $8 \times 8$ & $32 \times 32$ \\
\hline Pixel size & $5 \mathrm{~mm} \times 5 \mathrm{~mm}$ & $5 \mathrm{~mm} \times 5 \mathrm{~mm}$ & $1 \mathrm{~mm} \times 1 \mathrm{~mm}$ \\
\hline
\end{tabular}


Table 3: Hamamatsu MaPMT specifications:

\begin{tabular}{|l|l|}
\hline Parameter & Value \\
\hline Photocathode type & Bialkali \\
\hline Number of dynodes & 12 \\
\hline Total average gain @ -1kV & $\sim 10^{6}$ \\
\hline Geometrical collection efficiency of the 1-st dynode & $70-80 \%$ \\
\hline Geometrical packing efficiency & $97 \%$ \\
\hline Fraction of late photoelectron arrivals & $\sim 5 \%$ \\
\hline SLAC measurement of single electron resolution $\sigma_{\text {narrow }}$ & $138 \mathrm{ps}$ \\
\hline Amplifier used in SLAC measurement & Elantec $2075 \mathrm{C}$ \\
\hline Voltage gain of SLAC amplifier & $\sim 40 \mathrm{x}$ \\
\hline Matrix of anode pixels & $8 \times 8$ \\
\hline Pixel size & $5 \mathrm{~mm} \times 5 \mathrm{~mm}$ \\
\hline
\end{tabular}

(a)

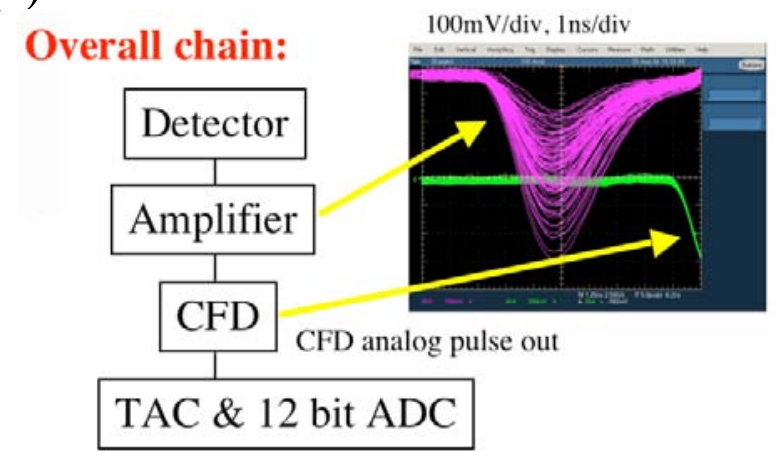

(b)

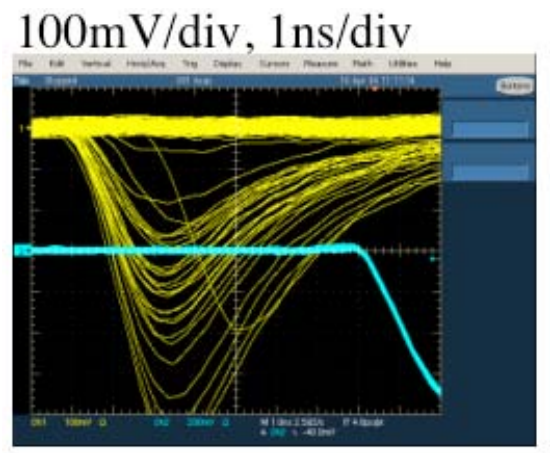

Fig. 6. (a) A block diagram of the Focusing DIRC prototype electronics built at SLAC. The amplifier is based on two Elantek 2075 chips connected in tandem with an overall gain of 130x and rise time of $\sim 1.5 \mathrm{~ns}$, followed by a constant fraction discriminator (CFD), followed by a TAC circuit and 12 bit ADC. The picture shows the amplifier output from the Burle MCP-PMT (S/N 85011-430) when the scope is triggered by a CFD analog output signal. (b) Amplifier output from the same tube, when scope is triggered with a PiLas light pulser (the detector is running in single photoelectron mode, and therefore, in this case, most of the traces are empty).

\section{TIMING MEASUREMENTS}

We have already shown in Ref. 6 that one can achieve good timing resolution performance with a few detector pixels. This paper shows the performance of much larger system with $\sim 300$ pixels. As one would expect, many things get correspondingly tougher. We have developed fast amplifiers based on a pair of two Elantek 2075 chips producing a voltage gain of 130x (this gain is needed for MCP-PMTs) with a $\sim 1.5 \mathrm{~ns}$ rise time. The constant-fraction discriminators (CFD) are coupled to a system of time-to-amplitude converters (TAC), which are coupled to 12-bit ADCs in turn, providing 25ps/count. This SLAC TDC system performs well. However, it was non-trivial to develop it, and it required a considerable amount of debugging/tweaking effort, especially the CFD/TAC part. To evaluate its performance, we have used commercial electronics such as LeCroy TDC2228, Philips TDC7186, Philips CFD715, etc. The most important part of the evaluation apparatus was the PiLas laser diode, ${ }^{6}$ which provides a timing resolution of $\sim 35$ ps FWHM. We would not be able to develop the system at all without this light source. Fig. 6 shows

\footnotetext{
6 PiLas laser diode is made by Advanced Laser Diode Systems, D-12489Berlin, Germany.
} 
examples of the amplifier output from the Burle MCP-PMT 85011-430 when the scope is triggered either by a CFD analog output signal, or the PiLas trigger.

Figure 7 shows the timing performance for two different 64-pixel Burle MCP-PMTs, and a Hamamatsu H-8500 Flat-panel MaPMTs. For example, Fig. 7c shows performance of the new MCP-PMT (\#16, S/N 02010410) which has a cathode-to-MCP distance of $0.75 \mathrm{~mm}$; this is to be compared with an older MCPPMT (\#3, S/N 05220304), which has this distance 6mm, giving much longer timing tail due to recoiling photoelectrons from the MCP top surface. Fig. $7 \mathrm{~d}$ shows an example of a single photon timing resolution across a single MCP-PMT (\#16) pad. One observes a deterioration of the resolution near the pad edges dues to the charge sharing and possible cross-talk. Figs. 7e\&f show examples of the performance of the 32channel CFD, using single photons obtained from the PiLas laser diode, and a 64-channel Burle MCP-PMT (\#16) with either double Gaussian fits, or single Gaussian fits. One can conclude that a typical single Gaussian fit yields $\sim 100 \mathrm{ps}$ on average. At the end, we will use a single Gaussian fit for the data analysis. At the time of writing this paper, about $90 \%$ of SLAC TDC channels performed as shown on Figs. 7e\&f, the remainder are slightly worse with single Gaussian fits indicating $\sigma \sim 120-140$ ps presently. However, this is still being studied and improvements are expected.

To study the ultimate timing resolution we can reach with the PiLas laser diode, we have decided to use two fast detectors: (a) a small GaP APD operating in a Geiger mode with active quenching [7], and (b) a single pixel MCP-PMT ${ }^{7}$ with $\sim 10 \mu \mathrm{m}$ MCP hole diameter. The single photoelectron timing distributions were fitted with a sum of two Gaussians plus a polynomial. The data indicate that the narrower Gaussian is: $\sigma_{\text {narrow }} \sim 38 \mathrm{ps}$ for the APD, and $\sigma_{\text {narrow }} \sim 33 \mathrm{ps}$ for the MCP-PMT (this value actually did not improve with the increasing number of photoelectrons, which indicates that we are already limited by some systematic error). The measured contribution of our electronics setup gives $\sigma_{\text {electronics }} \sim 15-20 \mathrm{ps}$, and the PiLas data sheets show that the laser diode light timing jitter relative to the trigger is $\sigma_{\text {PiLas }} \sim(35 / 2.35) \sim 15 \mathrm{ps}$, resulting in a total irreducible resolution of the setup of $\sigma \sim 25 \mathrm{ps}$. The resulting discrepancy is either due to a larger PiLas contribution compared to the company data sheets values, or some unknown additional systematic error. In any case, we feel that the present resolution limit with the PiLas laser diode operating in the single photon mode is somewhere in the range of $\sigma \sim 30-35 \mathrm{ps}$.

\footnotetext{
7 MCP-PMT (S/N 1448) was provided to us by A. Onuchin.
} 
(a)

Hamamatsu Flat Panel H8500 PMT:

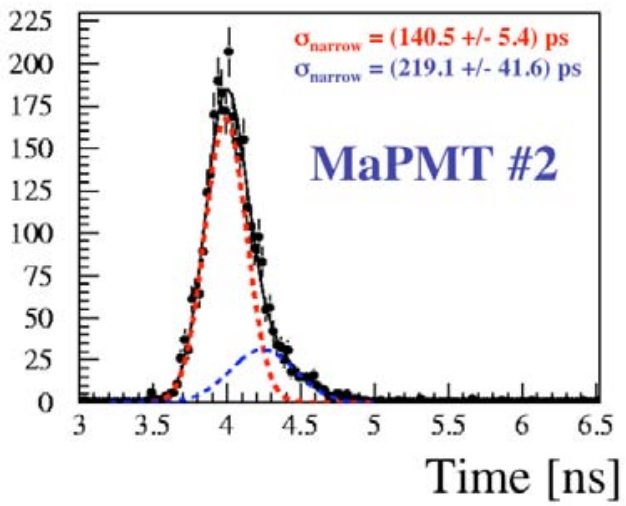

(c)

\section{Burle 85011-430 MCP-PMT:}

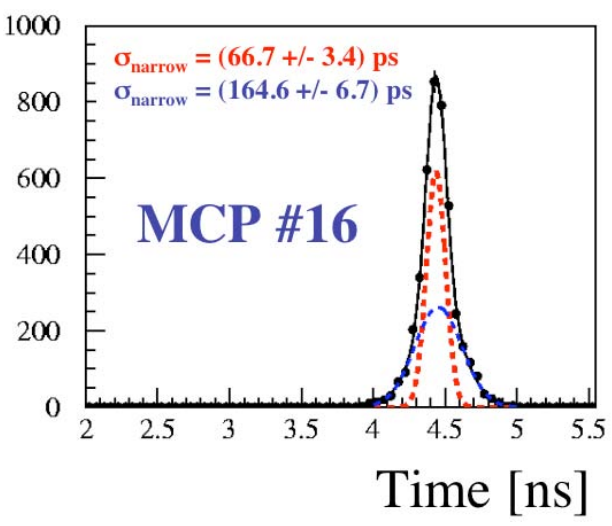

(e)

Double-Gaussian fits:

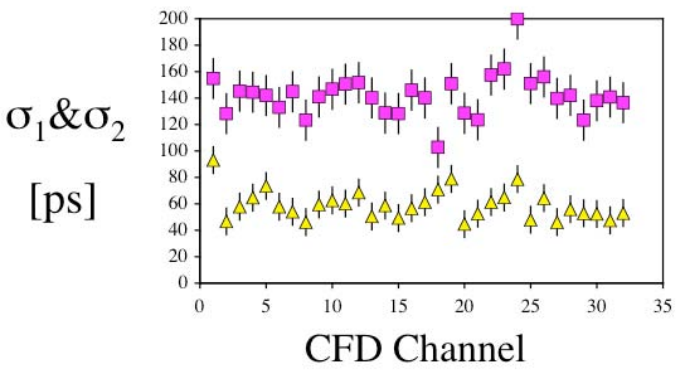

(b)

\section{Burle 85011-501 MCP-PMT:}

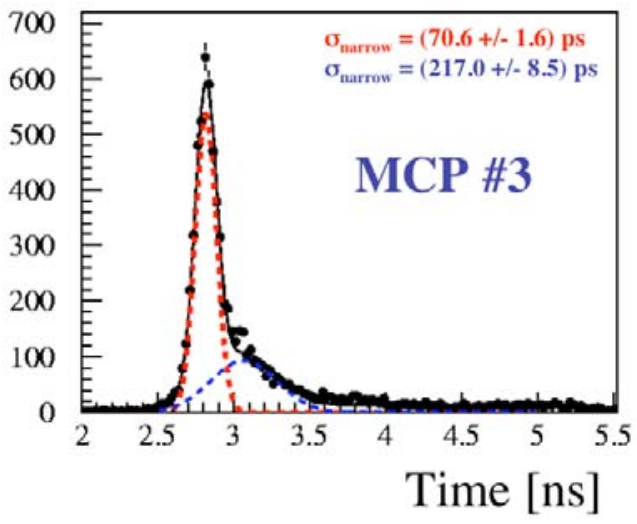

(d)

\section{Burle 85011-430 MCP-PMT, Pad 14:}

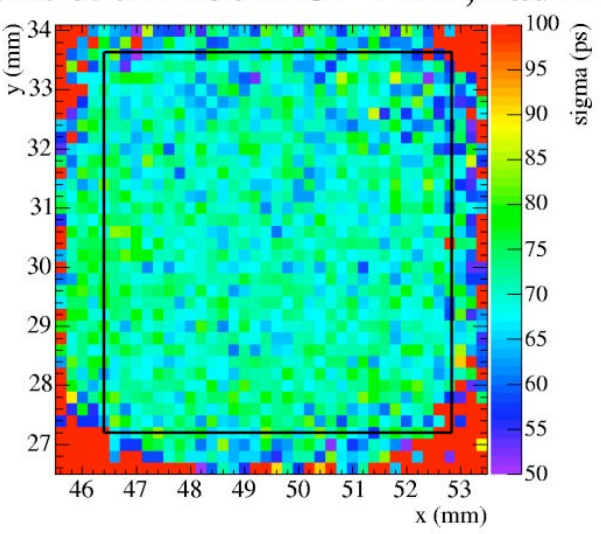

(f)

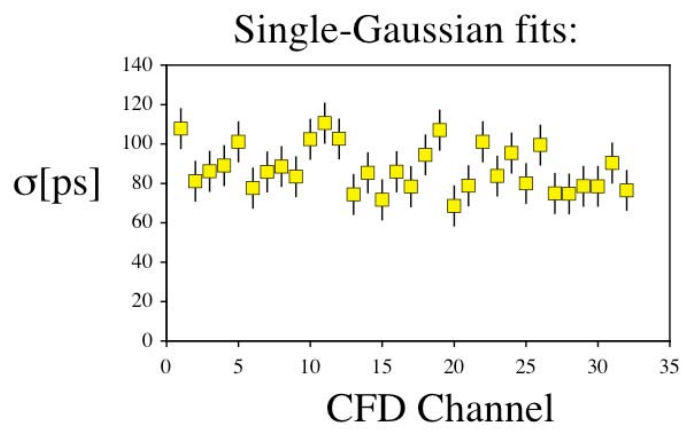

Fig. 7. Timing resolution obtained with the PiLas light pulser and (a) Hamamatsu MaPMT, (b) Burle MCP-PMT \#3 with a MCP-to-cathode distance of $7 \mathrm{~mm}$, (c) Burle MCP-PMT \#16 with a MCP-to-cathode distance of $0.7 \mathrm{~mm}$; (d) The same tube as (c), but showing a two-dimensional scan of the timing resolution across one pad; ${ }^{8}$ (e)\&(f) gives examples of fits across the 32 channels of CFD for the conditions is the same as (c); double Gaussian fits are characterized by narrow and wide Gaussian curves (e); single Gaussian fits to the same spectra (f).

\footnotetext{
8 Electronics in this particular measurement: a final SLAC amplifier, final SLAC 32-channel CFD providing the analog output to a Phillips 7186 TDC (25ps/count); the final SLAC TDC provides similar results.
} 
(a)

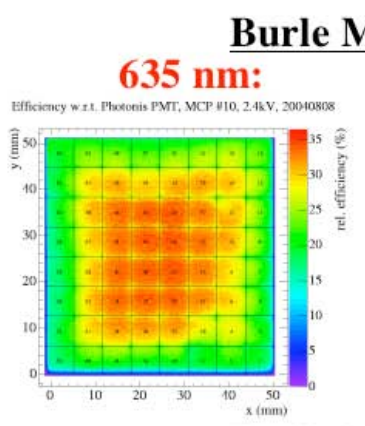

Burle MCP-PMT \#11

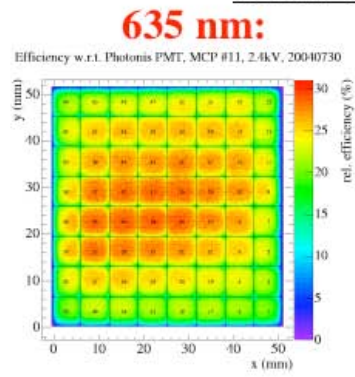

MCP-PMT \#10

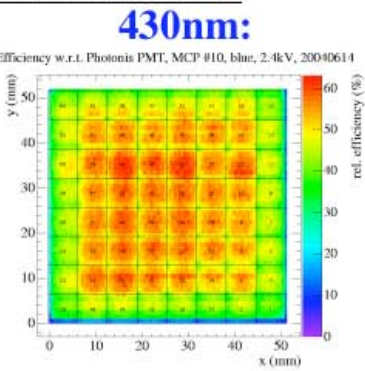

430nm:

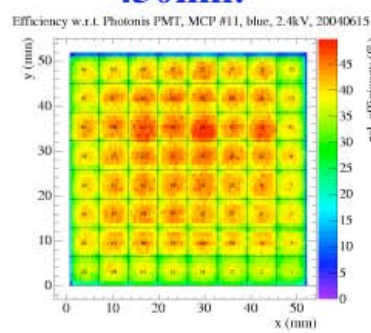

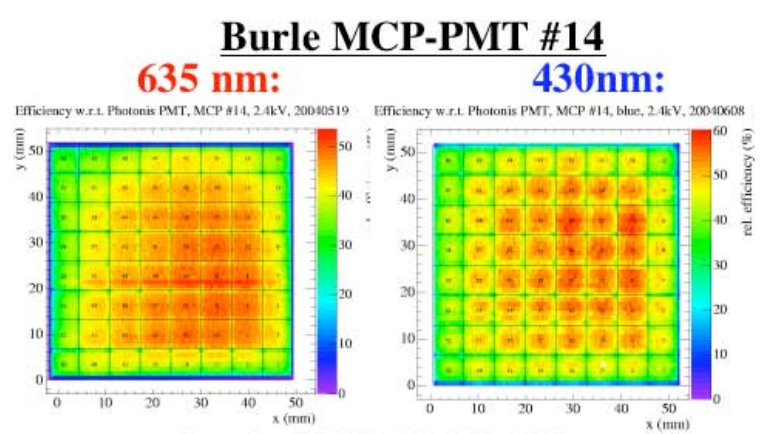

Burle MCP-PMT \#15
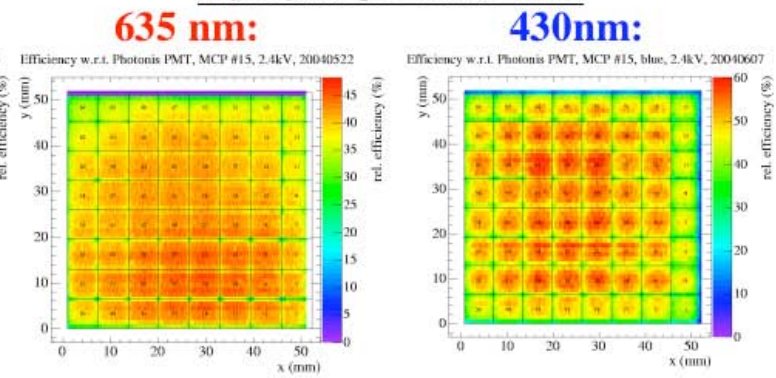

(b)
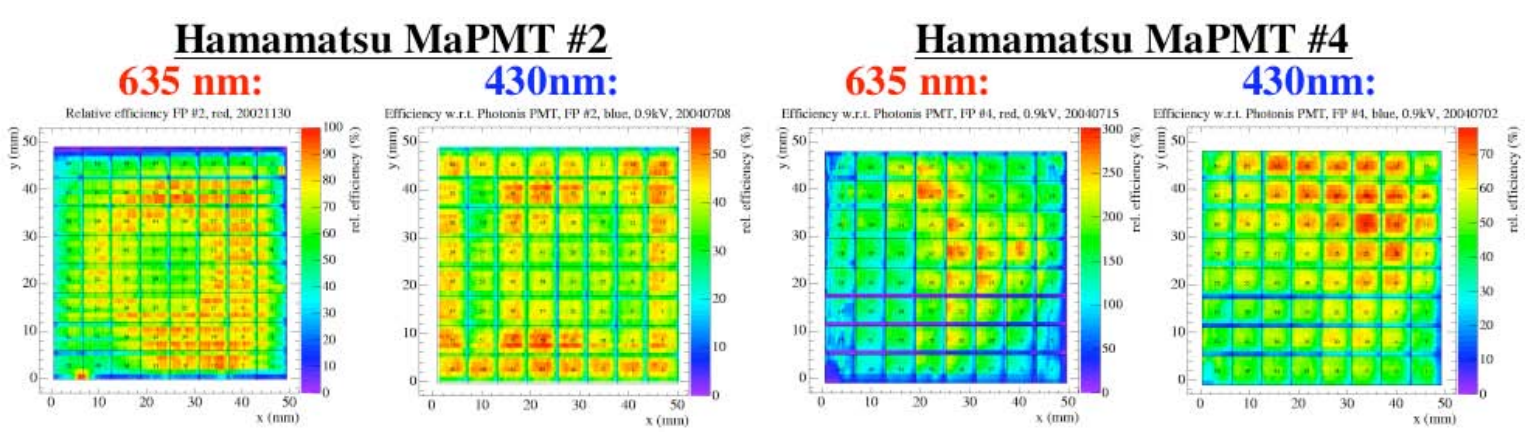

Fig. 8. Measurement of the relative single photoelectron response for: (a) Burle MCP-PMTs and (b) Hamamatsu MaPMTs. ${ }^{9}$ The measurement was done with the PiLas laser diode operating at two wavelengths 635 and $430 \mathrm{~nm}$.

\section{SPATIAL MEASUREMENTS}

Figure 8 shows the relative response across the faces of several 64-pixel photon detectors, such as the Burle MCP-PMT, or the Hamamatsu H-8500 Flat-panel MaPMT, using the PiLas laser diode operating in the single photoelectron mode at 635 or $430 \mathrm{~nm}$. The PiLas laser diode beam enters a 5-meter long $62.5 \mu \mathrm{m}$ multi-mode fiber via a lens and collimator. At the detector end, it exits the fiber again by a similar "lens $\&$ collimator" package, resulting in a beam spot of $\sim 150 \mu \mathrm{m}$. The fiber downstream end is mounted on a precision computer-controlled $\mathrm{x}-\mathrm{y}$ stage, providing small steps of typically $150 \mu \mathrm{m}$ in $\mathrm{x}$-direction and $1 \mathrm{~mm}$ in y direction. The scanning setup measures the relative response of the photon detector in the single photon mode using a $<1 \mathrm{~mm}$ spot size. The relative response measurement combines many contributions to the efficiency, such as the photocathode quantum efficiency, photoelectron transmission losses, detection

\footnotetext{
Electronics in this particullar measurement: a final SLAC amplifier, LeCroy 4413 discriminators with 100mV threshold, LeCroy 3377 TDCs with $0.5 \mathrm{~ns} /$ count; the final SLAC TDC provides similar results.
} 
efficiency, etc. A hit is accepted into the efficiency definition if it is within a time window, and it is on the same pad as the laser head is pointing to. To get a relative efficiency we normalize to the 2 inch dia. Photonis XP2262B PMT (or the DIRC PMT, ETL 9125). The electronics presently uses the final SLAC amplifier, CFD and TDC system. The laser diode power is adjusted so that the photodetector operates predominantly in the single photon counting mode, which is defined by requiring that the probability to get a single photoelectron is less than $10 \%$. The result of our studies of these two types of photon detectors indicate that the typical relative efficiency of the Burle MCP-PMT is 50-60\% of the 2 inch dia. Photonis XP 2262B PMT at $430 \mathrm{~nm}$, and the efficiency drops to $30-50 \%$ around the edges at $430 \mathrm{~nm}$. A typical relative efficiency of the Hamamatsu Flat Panel MaPMT is 50-70\% of the Photonis XP 2262B PMT at 430nm, and the efficiency drops to $30-50 \%$ around the edges at $430 \mathrm{~nm}$. Both tubes have edge effects where the efficiency drops by $\sim 10-20 \%$. The measured efficiencies at $430 \mathrm{~nm}$ are approximately consistent with expectations based on Tables $2 \& 3$. Fig. 8 also shows quite a large non-uniformity of the relative response across the face of the tubes. We find that the Hamamatsu MaPMT uniformity is typically $\sim 1: 2.5$, and the Burle MCPPMT uniformity is $\sim 1: 1.5$.

\section{AGING ISSUES}

We have started to investigate the MCP-PMT aging issues. This effect is well described the literature, and it is caused by the ion bombardment of the photocathode, i.e., it is related to quality of vacuum in the tube. As the vacuum scrubbing procedures improve, one hopes this problem gets reduced. According to Burle Co., one expects a 50\% photocathode degradation after an anode charge of $\sim 200 \mathrm{mC} / \mathrm{cm}^{2} .{ }^{10}$ Figure 9 shows a possible indication of the aging with an older four-pad Burle MCP-PMT (S/N 08290202) using large light pulses from the PiLas laser diode. Each pulse is equivalent of 270 photoelectrons. We observe a gain drop of 3-4\% after an accumulated dose of about $\sim 2 \times 10^{9}$ photoelectrons, which is equivalent to a $50 \%$ gain loss after a total charge of $\sim 200-250 \mathrm{mC} / \mathrm{cm}^{2}$. This is actually consistent with what the Burle Co. expects. More aging tests, especially with newer tubes, are needed to develop more definitive conclusions.

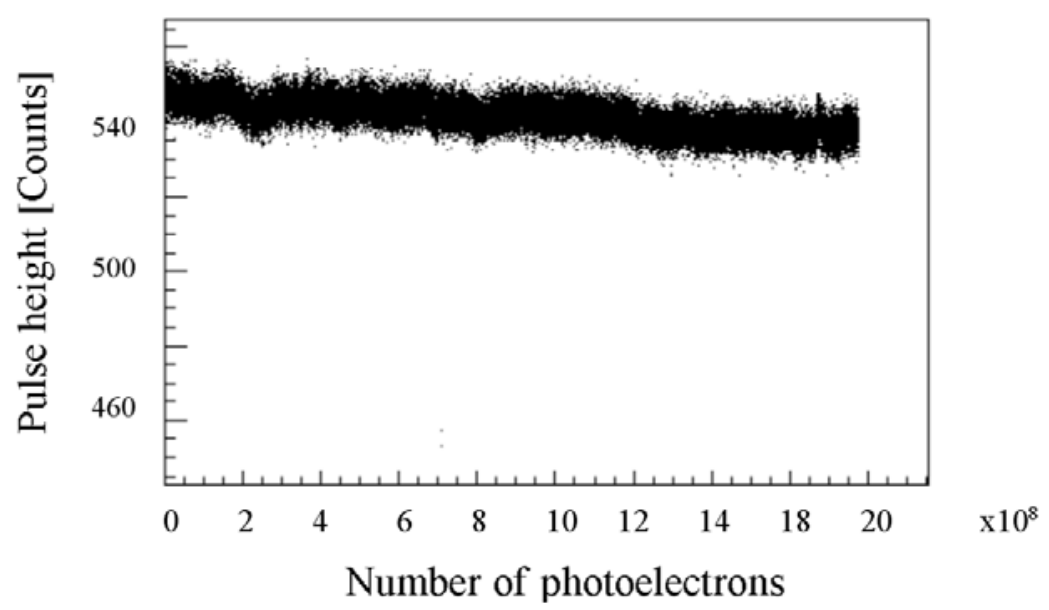

Fig. 9. Preliminary aging results with the older 2002 design four-pad Burle MCP-PMT using large light pulses from the PiLas laser diode. Each light pulse is equivalent of $\sim 270$ photoelectrons. We observe a gain drop of $\sim 3-4 \%$ after an accumulated dose of about $\sim 2 \times 10^{9}$ photoelectrons (note that the displayed quantity is the raw ADC value in counts of one MCP-PMT pad; the pedestal is 266 counts).

\footnotetext{
${ }^{10}$ According to Burle Co., the new versions of MCP-PMTs are supposed to be better by a factor of 2-3.
} 


\section{CONCLUSION}

This paper shows considerable progress towards the development of a new type of particle identification detector based on superior timing capability for each single Cherenkov photon. On a modest scale of a few hundred channels, we already have demonstrated the adequate timing resolution. On a scale of 25-30k pixels, which would be required for a "minimal" Focusing DIRC imaging system, we would clearly need further developments. One possible way to simplify the electronic design considerably is to go back to our original scheme, which was to use the double-threshold leading edge timing. This gives almost comparable timing resolution to the constant fraction discriminator, it is simpler, and it would allow a development of integrated electronics based on an ASIC design.

We have studied the spatial resolution of a number of MCP-PMTs and MaPMTs using a scanning setup with a spatial resolution of $\sim 150 \mu \mathrm{m}$. Many detailed microstructures are revealed, especially in the case of Flat Panel MaPMTs. The typical relative efficiency at 430nm, normalized to the Photonis PMT, is 50$60 \%$ for the Burle MCP-PMTs, and 60-70\% in good regions for the Hamamatsu Flat panel MaPMT. Both tubes have edge effects where the efficiency drops by $\sim 10-20 \%$.

The photon detectors need developments as well, especially to allow operation in a magnetic field, and to improve further aging performance. We believe that this paper represents a small step towards this goal.

\section{ACKNOWLEDGEMENT}

We would like to thank M. McCulloch and R. Reif for help to construct the prototype, and A. Scholz and T. Thurston for an engineering help. We thank Prof. G. Gratta for providing us with the KamLand experiment's mineral oil; Prof. T. Sumyioshi for letting us borrow his 430nm PiLas laser diode; and Prof. A. Onuchin for providing us with several of their single pixel MCP-PMTs.

\section{REFERENCES}

[1] I. Adam et al., "Particle Identification System for the BaBar experiment," Nucl. Instr.\&Meth., A538(2005)281-357.

[2] J. Schwiening, "Performance of the BABAR-DIRC," talk at this workshop, also SLAC-PUB-11017.

[3] B.N. Ratcliff, Nucl. Instr.\&Meth., A502(2003)211.

[4] J. Va'vra, "Cherenkov Imaging Techniques for the future High Luminosity Machines," Proc. of the 42-th Workshop of the INFN Eloisatron Project, September 2003, Erice, Italy, also SLAC-PUB-11019.

[5] Dolgoshein, "Silicon Photomultipliers in Particle Physics," Proc. of the 42-th Workshop of the INFN Eloisatron Project, September 2003, Erice, Italy.

[6] C. Field, T. Hadig, M. Jain, David W.G.S. Leith, G. Mazaheri, B. Ratcliff, J. Schwiening, and J. Va'vra, "Novel photon detectors for focusing DIRC prototype," Nucl. Instr. \& Meth., A518(2004)565-568.

[7] I. Prochazka et al., Optics Letters 17(1996)1375. 